\title{
Effect of Vitamin D3 on Biosynthesis of Estrogen in Porcine Granulosa Cells via Modulation of Steroidogenic Enzymes
}

\author{
So-Hye Hong ${ }^{1}$, Jae-Eon Lee ${ }^{1}$, Sung-Min An ${ }^{1}$, Ye Young Shin ${ }^{1}$, Dae Youn Hwang ${ }^{1}$, \\ Seung Yun Yang ${ }^{1}$, Seong-Keun $\mathrm{Cho}^{2+}$ and Beum-Soo $\mathrm{An}^{1+}$ \\ ${ }^{1}$ Departments of Biomaterials Science, ${ }^{2}$ Animal Science, College of Natural Resources \& Life Science, Pusan National University, Miryang, Korea
}

(Received November 15, 2016; Revised December 12, 2016; Accepted December 13, 2016)

\begin{abstract}
Vitamin D3 is a fat-soluble secosteroid responsible for enhancing intestinal absorption of calcium, iron, and other materials. Vitamin D3 deficiency, therefore, can cause health problems such as metabolic diseases, and bone disorder. Female sex hormones including estrogen and progesterone are biosynthesized mainly in the granulosa cells of ovary. In this study, we isolated granulosa cells from porcine ovary and cultured for the experiments. In order to examine the effect of vitamin D3 on the ovarian granulosa cells, the mRNA and protein levels of genes were analyzed by real-time PCR and Western blot assay. The production of estrogen from the granulosa cells was also measured by the ELISA assay. Genes associated with follicle growth were not significantly altered by vitamin D3. However, it increases expression of genes involved in the estrogen-biosynthesis. Further, estrogen concentrations in porcine granulosa cell-cultured media increased in response to vitamin D3. These results showed that vitamin D3 is a powerful regulator of sex steroid hormone production in porcine granulosa cells, suggesting that vitamin D deficiency may result in inappropriate sexual development of industrial animals and eventually economic loss.
\end{abstract}

Key words: Vitamin D3, Steroidogenesis, Follicle growth, Estrogen, Granulosa cell

Correspondence to: Beum-Soo An, Department of Biomaterials Science, College of Natural Resources \& Life Science, Pusan National University, 50 Cheonghak-ri, Samrangjin-eup, Miryang, Gyeongsangnam-do 50463, Korea

E-mail: anbs@pusan.ac.kr

Seong-Keun Cho, Department of Animal Science, Colledge of Natural Resources \& Life Science, Pusan National University, 50 Cheonghak-ri, Samrangjin-eup, Miryang, Gyeongsangnam-do 50463, Korea

E-mail: skcho@pusan.ac.kr

${ }^{\dagger}$ These authors contributed equally to this study.

Abbreviations: UV-B, ultraviolet B radiation; VD3, vitamin D3; VDR, vitamin $D$ receptor; $R X R$, retinoic acid receptor; VDRE, vitamin $D$ response element; $\mathrm{AMH}$, Anti-Mullerian hormone; FSHR, folliclestimulating hormone receptor; Foxl2, Forkhead box L2; StAR, steroidogenic acute regulatory protein; CYP11A1, side-chain cleavage enzyme; HSD3B1 3 $\beta$-hydroxysteroid dehydrogenase; CYP17A1, $17 \alpha$ hydroxylase; HSD17B1, $17 \beta$-hydroxysteroid dehydrogenase; CYP19A1, aromatase; E1, estrone; E2, estradiol; E3, estriol; E4, estetrol.

This is an Open-Access article distributed under the terms of the Creative Commons Attribution Non-Commercial License (http:// creativecommons.org/licenses/by-nc/3.0) which permits unrestricted non-commercial use, distribution, and reproduction in any medium, provided the original work is properly cited.

\section{INTRODUCTION}

Vitamin D, which comprises a group of fat-soluble secosterols found in very few foods, is photosynthesized in the skin of vertebrates by the action of solar ultraviolet $B$ radiation (UV-B) (1). The major biological function of vitamin D is the maintenance of normal concentrations of serum calcium and phosphorus by enhancing the efficiency of the small intestine to absorb these minerals from food (2). The original active form of vitamin $\mathrm{D}$ is derived from cholesterol. In the liver, cholecalciferol is converted into calcidiol. Part of calcidiol is converted by the kidneys into vitamin D3 (VD3), the biologically active form of vitamin D $(3,4)$. VD3 circulates as a hormone in the blood, mediating physiological functions such as calcium absorption (5). According to previous studies, a low level of vitamin D is associated with immune disease, cardiovascular disease, diabetes, and obesity (6-11). VD3 mediates its biological effects by binding to vitamin D receptor (VDR), which is mainly located in the nuclei of target cells in complex with 9-cis-retinoic acid receptor (RXR). The activated VDRRXR complex binds to a vitamin $\mathrm{D}$ response element (VDRE) located in the promoters of target genes (12). VDR 
is expressed by cells in most organs, including the brain, heart, intestine, and skin. Reproductive organs such as the prostate, breast, placenta, uterus, and ovaries also express VDR, whereas the physiological roles of VDR in these organs are not clear (13-16).

The ovaries are a female reproductive organ consisting of granulosa and theca cells. Granulosa cells surround oocytes and produce female sex steroid hormones, including progesterone and estrogen (17). Follicle growth is based on oocytes and granulosa cells of the follicle in early growth stages, and steroidogenesis is accelerated by follicle development $(18,19)$. Anti-Mullerian hormone (AMH) and follicle-stimulating hormone receptor (FSHR) are known as representative biomarkers in folliculogenesis $(20,21)$. Forkhead box L2 (Foxl2) also plays an important role in follicle growth and ovarian differentiation (22). Steroidogenesis is the pathway of steroid hormone biosynthesis and begins with cholesterol as the substrate. The first step of steroidogenesis is mediated by steroidogenic acute regulatory protein (STAR) via transportation of cholesterol into mitochondria. Side-chain cleavage enzyme (CYP11A1) converts cholesterol into pregnenolone, which can be converted into progesterone by $3 \beta$-hydroxysteroid dehydrogenase (HSD3B1). Another important female sex steroid hormone, estrogen, is synthesized by $17 \alpha$ hydroxylase (CYP17A1), 17ß-hydroxysteroid dehydrogenase (HSD17B1), and aromatase (CYP19A1) from cholesterol, pregnenolone, $17 \alpha-$ hydroxy progesterone, androstendione, and testosterone (19,23).

Estrogen is the primary female sex hormone and is responsible for development and regulation of the female reproductive system and secondary sex characteristics (24). The three major naturally occurring forms of estrogen in women are estrone (E1), estradiol (E2), and estriol (E3). Another type of estrogen called estetrol (E4) is produced only during pregnancy (25). The steroid $17 \beta$-estradiol is the most potent and prevalent type of endogenous estrogen, although several metabolites of E2 also have estrogenic hormonal activity.

Although estrogen biosynthesis is clearly related with maturation of industrial animals, only a few studies have made this issue a focus. In addition, the effects of environmental circumstances such as sunlight exposure on sexual maturation have not been addressed in pigs. In this study, we examined the effects of vitamin D on folliculogenesis and biosynthesis of estrogen in porcine granulosa cells.

\section{MATERIAL AND METHODS}

Primary culture of granulosa cells. Granulosa cells were isolated from $3 \sim 5 \mathrm{~mm}$-sized follicles of immature pigs. Cells were washed three times by centrifugation (1500 rpm) in phosphate-buffered saline (PBS) containing 1\% streptomycin/penicillin (Welgene Inc., Seoul, Korea). Granulosa cells were cultured in DMEM (Gibco-BRL, Gaithersburg, MD, USA) supplemented with $10 \%$ fetal bovine serum (FBS, Gibco-BRL) and 1\% streptomycin/penicillin. Incubation of the granulosa cell suspension was carried out in 6-well dishes at $37^{\circ} \mathrm{C}$ in an atmosphere of $5 \% \mathrm{CO}_{2}-95 \%$ air. Cell density and viability were determined in a hemocytometer by trypan blue exclusion. Cell viability ranged from $70 \%$ to $80 \%$. After $24 \mathrm{hrs}$, medium was exchanged to remove red blood cells. After incubation for $48 \mathrm{hrs}$, medium was replaced with phenol-free DMEM supplemented with $10 \%$ charcoal-dextran-treated FBS and 1\% streptomycin/ penicillin $24 \mathrm{hrs}$ before treatment. VD3 (Millipore, MA, USA) was administered at a concentration of $100 \mathrm{nM}$ (26), and an equal part of ethanol was added to the control group.

Quantification of mRNA by real-time PCR. Total RNA was extracted using TRIzol reagent (Invitrogen Co., Carlsbad, CA, USA) according to the manufacturer's protocol. The concentration of total RNA was measured by a BioSpec-nano (Bio-Rad, CA, USA). Complementary DNA

Table 1. Primer sequences for Q-PCR analysis

\begin{tabular}{lllc}
\hline \hline Gene name & Primer & \multicolumn{1}{c}{ Sequence (5'- 3') } & Fragment size (bp) \\
\hline \multirow{2}{*}{-Actin } & Forward & TCC CTG GAG AAG AGC TAC GA & 249 \\
& Reverse & CGC ACT TCA TGA TCG AGT TG & 220 \\
AMH & Forward & GTG GTG GGG ACT CTA AGC AG & 214 \\
& Reverse & CTC CTG GAA CTT CAG CAA GG & 160 \\
FSHR & Forward & ATG TCC TTG CTC CTG GTG TC & \\
& Reverse & GGT CCC CAA ATC CAG AAA AT & 188 \\
Fox12 & Forward & ACA TGT TCG AGA AGG GCA AC & 243 \\
CYP17A1 & Reverse & GGT AGT GGC CAC GAG TTG TT & \\
& Forward & CCA AGG AGG TGC TTC TCA AG & 243 \\
HSD17B1 & Reverse & GTT CTC CAG CTT CAG GTT GC & \\
& Forward & GGC TTT TGC AGA AAG AGG TG & \\
\hline
\end{tabular}


(cDNA) was prepared from total RNA $(2 \mu \mathrm{g})$ by reverse transcription (RT) using M-MLV reverse transcriptase (Invitrogen Co.) and random primers (9-mers; TaKaRa Bio Inc., Shiga, Japan). Q-PCR was performed using cDNA template $(2 \mu \mathrm{L})$ and SYBR Green $(6 \mu \mathrm{L}$; TOYOBO Co., Ltd., Katata, Ohtsu, Japan) containing specific primers. The primer sequences for AMH, FSHR, CYP17A1, HSD17B1, and CYP19 are shown in Table 1. Q-PCR was carried out for 40 cycles using the following parameters: denaturation at $95^{\circ} \mathrm{C}$ for $15 \mathrm{~s}$, annealing, and extension at $70^{\circ} \mathrm{C}$ for $60 \mathrm{~s}$. Fluorescence intensity was measured at the end of each extension phase. The threshold value for the fluorescence intensities of all samples was set manually. The reaction cycle at which PCR products exceeded this fluorescence intensity threshold during the exponential phase of PCR amplification was considered to be the cycle of threshold (CT). Expression of the target gene was quantified relative to that of $\beta$-actin, a housekeeping gene, based on comparison of CTs at a constant fluorescence intensity.

Western blot analysis. Protein was extracted with Proprep (Intron Co., Seoul, South Korea) following the manufacturer's protocol. Cytosolic protein $(30 \mu \mathrm{g})$ was subjected to $10 \%$ to $12 \%$ SDS-PAGE and transferred to a nitrocellulose membrane (Daeillab Service Co., Ltd, Seoul, South Korea). The membranes were then blocked for $2 \mathrm{hr}$ with $5 \%$ skim milk (Difco ${ }^{\mathrm{TM}}$, Sparks, MD, USA) or 5\% donkey serum (Sigma-Aldrich Inc., St. Louis, MO, USA) in PBS with $0.05 \%$ Tween-20 (PBS-T). After blocking, membranes were incubated with antibodies specific for CYP19A1 (diluted 1:500) and CYP17A1 (diluted 1:500) overnight as well as HRP-conjugated anti-goat and anti-rabbit secondary antibodies (diluted 1:2000) in blocking buffer with PBS-T for $1 \mathrm{hr}$. Luminol reagent (Bio-Rad Laboratories Inc., Hercules, CA, USA) was used to visualize binding of antibodies. Each blot was then stripped by incubation with $2 \%$ SDS and $100 \mathrm{mM}$ mercaptoethanol in $62.5 \mathrm{mM}$ Tris$\mathrm{HCl}\left(\mathrm{pH} 6.8\right.$ ) for $30 \mathrm{~min}$ at $50 \sim 60^{\circ} \mathrm{C}$. Membranes were subsequently probed with antibody against $\beta$-actin (diluted
1:3000, Santa Cruz Biotechnology) as an internal control. Blots were then scanned using Gel Doc 1000, version 1.5 (Bio-Rad Laboratories Inc.), and band intensities were normalized to $\beta$-actin levels.

Measurement of E2. After VD3 treatments, cultured media were centrifuged at $1300 \mathrm{~g}$ for $15 \mathrm{~min}$ and stored at $-80^{\circ} \mathrm{C}$. E2 accumulation in media was measured using a competitive enzyme immunoassay kit (Cayman Chemical, Ann Arbor, MI, USA) following the manufacturer's protocol.

Statistical analysis. Results are presented as the mean \pm standard deviation (SD). Data were analyzed for statistical significance using Sigma Plot 10.0 followed by $t$-test (Software Inc., San Jose, CA, USA). $P$-values $<0.05$ were considered statistically significant.

\section{RESULTS}

Regulation of folliculogenesis-related genes in porcine granulosa cells. As follicles develop, production of female hormones such as progesterone and estrogen production increases. To examine how VD3 regulates follicular growth, expression levels of genes associated with folliculogenesis, including FSHR, AMH, and FOXL2, were measured in porcine ovarian granulosa cells. The mRNA expression levels of these genes were analyzed by real-time PCR. Treatment of granulosa cells with VD3 up-regulated expression of all tested genes, although the results were not significantly different compared with the control (Fig. 1).

Regulation of estrogen biosynthesis-related genes in porcine granulosa cells. To examine biosynthesis of E2, the mRNA expression levels of CYP17A1, HSD17B1, and CYP19A1 enzymes were evaluated (Fig. 2). Expression of these genes was significantly enhanced upon VD3 treatment for $24 \mathrm{hr}$. To confirm these findings, protein levels of CYP17A1, HSD17B1, and CYP19A1 were tested (Fig. 3). In our study, HSD17B1 was chosen since this isoform is
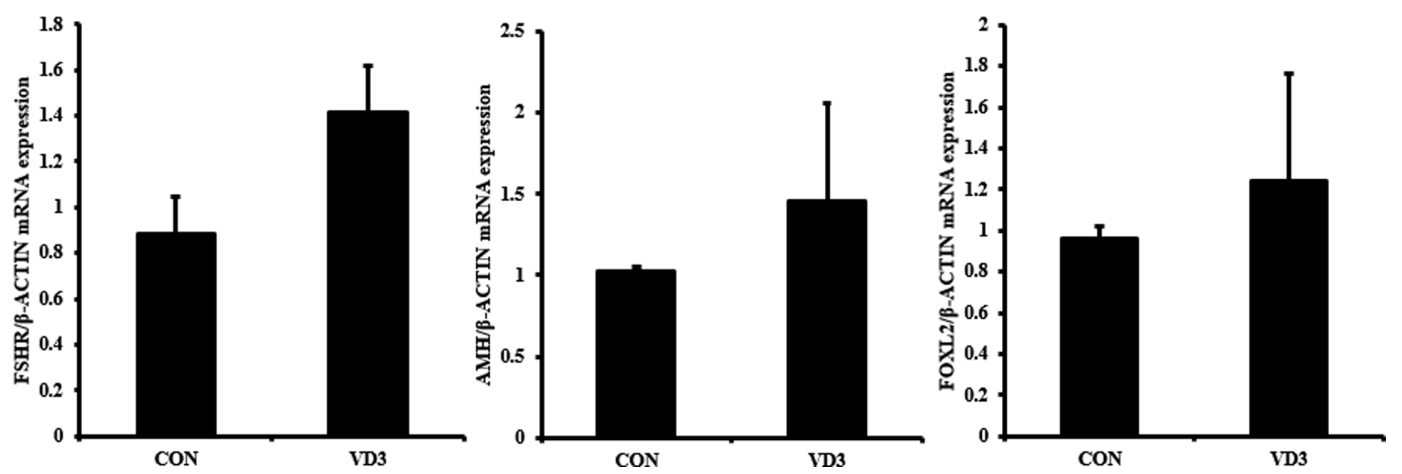

Fig. 1. The mRNA expression of folliculogenic enzymes in granulosa cells treated with VD3. AMH, FSHR, and Fox/2 expression levels were analyzed by real-time PCR. Gene expression levels were normalized to the level of $\beta$-actin. Data are expressed as the mean \pm SD. 


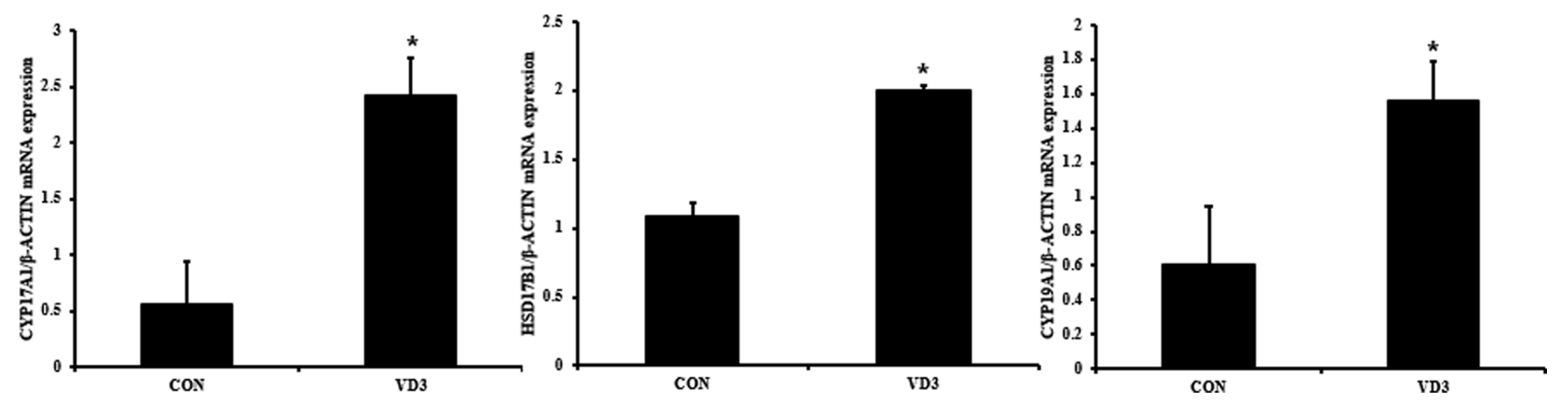

Fig. 2. The mRNA expression of E2 biosynthesis-related enzymes in granulosa cells treated with VD3. CYP17A1, HSD17B1, and CYP19A1 expression levels were analyzed by real-time PCR. Gene expression levels were normalized to the level of $\beta$-actin. Data are expressed as the mean $\pm \mathrm{SD}$. ${ }^{*} P<0.05$ compared to control group.
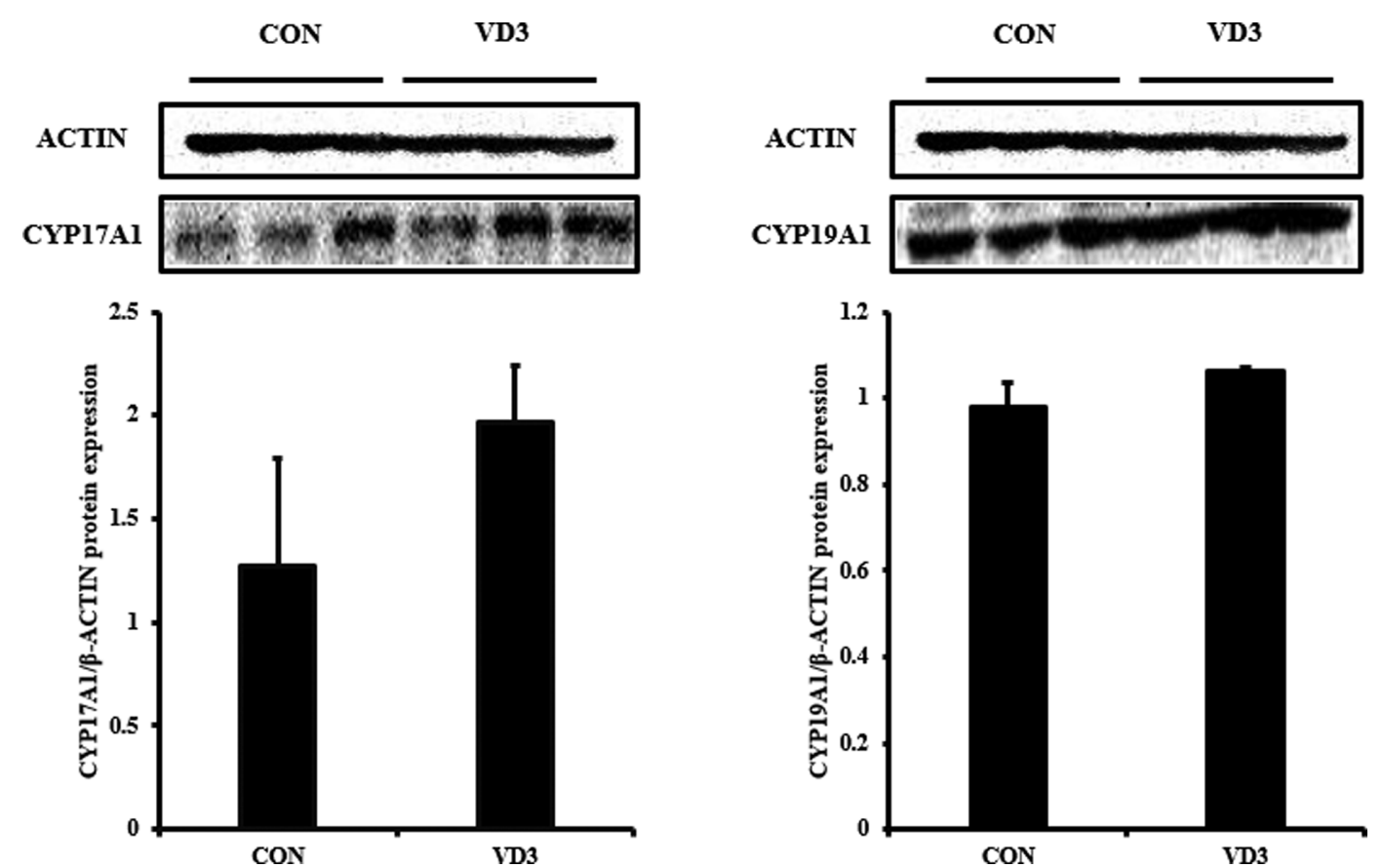

Fig. 3. The protein expression of E2 biosynthesis-related proteins in granulosa cells treated with VD3. CYP17A1 and CYP19A1 levels were analyzed by Western blot assay. Protein levels were normalized by $\beta$-actin. Data are expressed as the mean \pm SD.

known to be expressed in the ovaries. However, the signal for HSD17B1 was not detected in porcine granulosa cells (data not shown). Protein expression of CYP17A1 and CYP19A1 tended to increase, although the results were not significant.

Analysis of estrogen levels after VD3 treatment. Since the mRNA expression of E2 biosynthesis-related enzymes was significantly altered by VD3, we examined E2 secretion in granulosa cell-cultured media (Fig. 4). Basal E2 level in granulosa cell-cultured media collected from the control group was $0.18 \mathrm{ng} / \mathrm{mL}$. Consistent with the results of E2-metabolizing enzymes, VD3 increased production of E2 in cultured media up to 3 -fold compared with the control.

\section{DISCUSSION}

E2 regulates the menstrual cycle, controlling the growth of the uterine lining during the first part of the cycle (27). E2 also involved in the onset of puberty, playing a role in the development of secondary sex characteristics. Due to these functions, E2 is related with many reproductive diseases, including abortion, stillbirth, endometritis, ovarian cancer, and breast cancer $(28,29)$. Particularly, reproductive diseases in industrial animals such as pigs, cows, and chickens are of critical concern since these animals are a main source of animal meat for human consumption. Current research on the reproductive systems of industrial animals is directly focused on increasing litter size and fertility rate. 


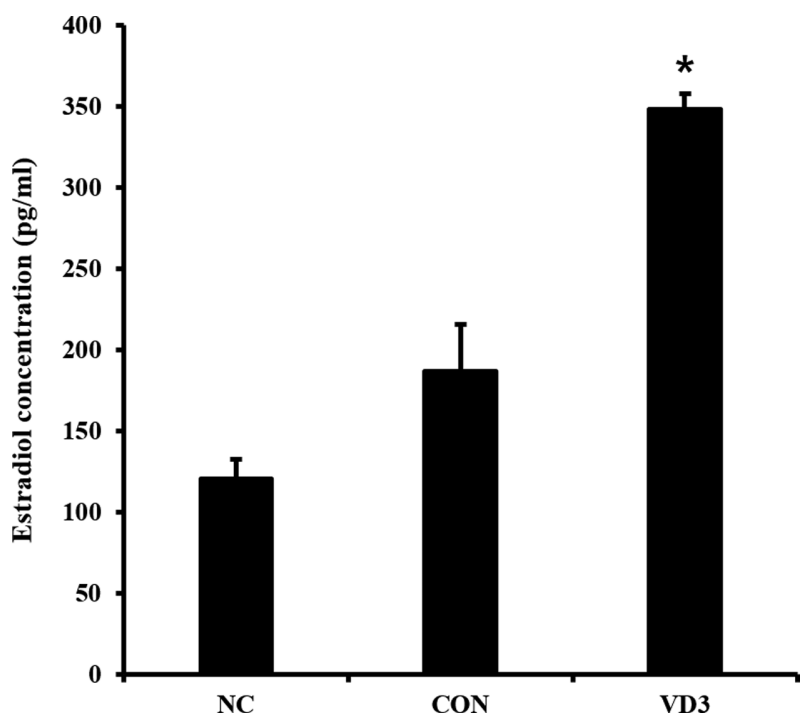

Fig. 4. Effects of VD3 on E2 secretion in granulosa cells. After treatment of granulosa cells with VD3, medium was harvested. E2 concentration in cell growth medium was analyzed by ELISA assay. NC, negative control, media without granulosa cells; Con, control media with granulosa cells. Data are expressed as the mean \pm SD. ${ }^{*} P<0.05$ compared to control group.

Despite these issues, most studies on reproduction in animals have been carried out in rodents due to difficulties in sample collection. Many endogenous and exogenous factors can influence production of E2 and sexual maturation. For example, hormones such as FSH, LH, GnRH, inhibin, and activin are all known to regulate E2 synthesis (30). Exogenous circumstances such as external stress, air, water intake, and food may additionally regulate production of E2. In addition, exposure to sunlight and VD3 production can affect E2 biosynthesis. Since pigs are often reared in conditions lacking sunlight, such as a pigsty, the effects of sunlight and VD3 on E2 synthesis and sexual development should be investigated.

In this study, we examined the effect of VD3 on folliculogenesis and biosynthesis of E2 in porcine granulosa cells. Although VD3 did not significantly alter expression of genes involved in follicular development, expression of steroidogenic enzymes, including CYP17A1, HSD17B1, and CYP19A1, was affected in other species. Previously, it was shown that VD3 regulates AMH and FSHR mRNA expression in granulosa cells from chickens (31). Specifically, VD3 was shown to down-regulate $\mathrm{AMH}$ and up-regulate FSHR gene expression in hen ovaries, whereas steroidogenesis-related enzymes were not tested (31). In human granulosa cells, VD3 does not significantly regulate expression of steroidogenic enzymes (32). The difference between our and previous results is may be because of a species specific regulation of the genes by VD3.

In the present study, we examined the regulatory effects of VD3 on E2 synthesis in granulosa cells and found that VD3 significantly augmented production of E2. In previous studies, VD3 increased E2 and progesterone biosynthesis in mice, although these studies did not analyze expression of steroidogenic enzymes. We examined the effects of VD3 on folliculogenesis and E2 biosynthesis in porcine granulosa cells. Our results demonstrate that VD3 is a powerful modulator of E2 production, although follicular growth was not affected. These findings suggest that sunlight exposure and enhanced VD3 production in pigs may regulate E2 production and promote sexual development, resulting in an increased birth rate.

\section{ACKNOWLEDGMENTS}

This work was supported by a 2-Year Research Grant of Pusan National University.

\section{REFERENCES}

1. Holick, M.F. (1994) McCollum Award Lecture, 1994: vitamin D--new horizons for the 21 st century. Am. J. Clin. Nutr., 60, 619-630.

2. Bell, T.D., Demay, M.B. and Burnett-Bowie, S.A. (2010) The biology and pathology of vitamin $\mathrm{D}$ control in bone. J. Cell. Biochem., 111, 7-13.

3. Hollis, B.W. (1996) Assessment of vitamin D nutritional and hormonal status: what to measure and how to do it. Calcif. Tissue Int., 58, 4-5.

4. DeLuca, H.M., Holick, M.F., Schnoes, H.K., Suda, T. and Cousins, R.J. (1971) Isolation and identification of 1, 25-dihydroxycholecalciferol. A metabolite of vitamin D active in intestine. Biochemistry, 10, 2799-2804.

5. DeLuca, H.F. (2004) Overview of general physiologic features and functions of vitamin D. Am. J. Clin. Nutr., 80, 1689S-1696S.

6. Hewison, M. (2008) Vitamin D and innate immunity. Curr. Opin. Investig. Drugs, 9, 485-490.

7. Hart, P.H. (2012) Vitamin D supplementation, moderate sun exposure, and control of immune diseases. Discov. Med., 13, 397-404.

8. Ponsonby, A.L., McMichael, A. and van der Mei, I. (2002) Ultraviolet radiation and autoimmune disease: insights from epidemiological research. Toxicology, 181,71-78.

9. Bolland, M.J., Grey, A., Gamble, G.D. and Reid, I.R. (2014) The effect of vitamin D supplementation on skeletal, vascular, or cancer outcomes: a trial sequential meta-analysis. Lancet Diabetes Endocrinol., 2, 307-320.

10. Forman, J.P., Scott, J.B., Ng, K., Drake, B.F., Suarez, E.G., Hayden, D.L., Bennett, G.G., Chandler, P.D., Hollis, B.W., Emmons, K.M., Giovannucci, E.L., Fuchs, C.S. and Chan, A.T. (2013) Effect of vitamin D supplementation on blood pressure in blacks. Hypertension, 61, 779-785.

11. Kang, E.J., Lee, J.E., An, S.M., Lee, J.H., Kwon, H.S., Kim, B.C., Kim, S.J., Kim, J.M., Hwang, D.Y., Jung, Y.J., Yang, S.Y., Kim, S.C. and An, B.S. (2015) The effects of vitamin D3 on lipogenesis in the liver and adipose tissue of pregnant rats. 
Int. J. Mol. Med., 36, 1151-1158.

12. Pike, J.W., Meyer, M.B. and Martowicz, M.L. (2009) New techniques in transcription research extend our understanding of the molecular actions of the vitamin D hormone. IBMS BoneKEy, 6, 169-180.

13. Zhang, R. and Naughton, D.P. (2010) Vitamin D in health and disease: current perspectives. Nutr. J., 9, 65 .

14. Zarnani, A.H., Shahbazi, M., Salek-Moghaddam, A., Zareie, M., Tavakoli, M., Ghasemi, J., Rezania, S., Moravej, A., Torkabadi, E., Rabbani, H. and Jeddi-Tehrani, M. (2010) Vitamin D3 receptor is expressed in the endometrium of cycling mice throughout the estrous cycle. Fertil. Steril., 93, 2738-2743.

15. Avila, E., Díaz, L., Halhali, A. and Larrea, F. (2004) Regulation of 25-hydroxyvitamin D $31 \alpha$-hydroxylase, 1, 25-dihydroxyvitamin D3 24-hydroxylase and vitamin D receptor gene expression by 8-bromo cyclic AMP in cultured human syncytiotrophoblast cells. J. Steroid Biochem. Mol. Biol., 89, 115119.

16. Irani, M. and Merhi, Z. (2014) Role of vitamin D in ovarian physiology and its implication in reproduction: a systematic review. Fertil. Steril., 102, 460-468.

17. Kotsuji, F. and Tominaga, T. (1994) The role of granulosa and theca cell interactions in ovarian structure and function. Microsc. Res. Tech., 27, 97-107.

18. Eppig, J.J., Wigglesworth, K. and Pendola, F.L. (2002) The mammalian oocyte orchestrates the rate of ovarian follicular development. Proc. Natl. Acad. Sci. U.S.A., 99, 2890-2894.

19. Payne, A.H. and Hales, D.B. (2004) Overview of steroidogenic enzymes in the pathway from cholesterol to active steroid hormones. Endocr. Rev., 25, 947-970.

20. Peluso, C., Fonseca, F.L., Rodart, I.F., Cavalcanti, V., Gastaldo, G., Christofolini, D.M., Barbosa, C.P. and Bianco, B. (2014) AMH: An ovarian reserve biomarker in assisted reproduction. Clin. Chim. Acta, 437, 175-182.

21. Tao, Y.X. and Segaloff, D.L. (2009) Follicle stimulating hormone receptor mutations and reproductive disorders. Prog.
Mol. Biol. Transl. Sci., 89,115-131.

22. Georges, A., Auguste, A., Bessière, L., Vanet, A., Todeschini, A.L. and Veitia, R.A. (2013) FOXL2: a central transcription factor of the ovary. J. Mol. Endocrinol., 52, R17-R33.

23. Hanukoglu, I. (1992) Steroidogenic enzymes: structure, function, and role in regulation of steroid hormone biosynthesis. $J$. Steroid Biochem. Mol. Biol., 43, 779-804.

24. Zhu, B.T. and Conney, A.H. (1998) Functional role of estrogen metabolism in target cells: review and perspectives. Carcinogenesis, 19, 1-27.

25. Gruber, C.J., Tschugguel, W., Schneeberger, C. and Huber, J.C. (2002) Production and actions of estrogens. N. Engl. J. Med., 346, 340-352.

26. Hong S.H., Lee, J.E., Kim, H.S., Jung, Y.J., Hwang, D., Lee, J.H., Yang, S.Y., Kim, S.C., Cho, S.K. and An, B.S. (2016) Effect of vitamin D3 on production of progesterone in porcine granulosa cells by regulation of steroidogenic enzymes. J. Biomed. Res., 30, 203-208.

27. Brody, S. and Wiqvist, N. (1961) Ovarian hormones and uterine growth: Effects of estradiol and progesterone on cell growth and cell division in the rat uterus. Endocrinology, 68, 971-977.

28. Okada, H. (1993) Metabolism, structure and biological activity of sex steroids. Nihon Naibunpi Gakkai Zasshi, 69, 67-79.

29. Distler, W. (1993) The role of sex steroids for genesis of breast and endometrial cancer. Gynakologe, 26, 94-99.

30. Yoshiki, N. and Aso, T. (1997) The regulation mechanism of the female menstrual cycle. Nippon Rinsho, 55, 2840-2848.

31. Wojtusik, J. and Johnson, P.A. (2012) Vitamin D regulates anti-Mullerian hormone expression in granulosa cells of the hen. Biol. Reprod., 86, 91.

32. Merhi, Z., Doswell, A., Krebs, K. and Cipolla, M. (2014) Vitamin D alters genes involved in follicular development and steroidogenesis in human cumulus granulosa cells. J. Clin. Endocrinol. Metab., 99, E1137-E1145. 